\title{
Approximate pole-placement controller using inverse plant dynamics for floor vibration control
}

\author{
Donald S. Nyawako*a, Paul Reynolds, ${ }^{\mathrm{a}, \mathrm{b}}$, Malcolm J. Hudson ${ }^{\mathrm{a}}$ \\ ${ }^{a}$ The University of Sheffield, Department of Civil and Structural Engineering, Sheffield, UK; \\ ${ }^{\mathrm{b}}$ Full Scale Dynamics Limited, UK
}

\begin{abstract}
Past research and field trials have demonstrated the viability of active vibration control (AVC) technologies for the mitigation of human induced vibrations in problematic floors. They make use of smaller units than their passive counterparts, provide quicker and more efficient control, can tackle multiple modes of vibration simultaneously and adaptability can be introduced to enhance their robustness. Predominantly single-input-single-output (SISO) and multiSISO collocated sensor and actuator pairs have been utilized in direct output feedback schemes, for example, with direct velocity feedback (DVF). On-going studies have extended such past works to include model-based control approaches, for example, pole-placement (PP), which demonstrate increased flexibility of achieving desired vibration mitigation performances but for which stability issues must be adequately addressed.

The work presented here is an extension to the pole-placement controller design using an algebraic approach that has been investigated in past studies. An approximate pole-placement controller formulated via the inversion of the floor dynamics, considered as minimum phase, is designed to achieve target closed-loop performances. Analytical studies and experimental tests are based on a laboratory structure and comparisons in vibration mitigation performances are made with a typical DVF control scheme with inner loop actuator compensation. It is shown that with minimal compensation, primarily in the form of notch filters and gain adjustment, the approximate pole-placement controller scheme is easily formulated and implemented and offers good vibration mitigation performance as well as the potential for isolation and control of specific target modes of vibration. Predicted attenuations of $22 \mathrm{~dB}$ and $12 \mathrm{~dB}$ in both the first and second vibration modes of the laboratory structure were also realized in the experimental studies for DVF and the approximate PP controller.
\end{abstract}

Keywords: active vibration control, direct velocity feedback, pole-placement

\section{INTRODUCTION}

As a result of advancements in design and construction technologies and changes in typical fit-outs, flexible floor structures that are more prone to human-induced vibrations are becoming more common. Whilst there are various vibration mitigation techniques that can be pursued as remedial measures when such vibration serviceability problems arise, additional emphasis should also be placed on the potential incorporation of vibration mitigation techniques into the mainstream design process of floors when it is anticipated such problems may arise. This could result in more efficient and high quality floor structures with enhanced vibration performance.

Past research and field trials have demonstrated the viability of active vibration control (AVC) technologies for the mitigation of human induced vibrations in problematic floors using combinations of direct output feedback (DOFB) and model-based controllers ${ }^{[1,2,3,4,5,6]}$. They make use of smaller units than some of their passive counterparts, provide quicker and more efficient control, can tackle multiple modes of vibration simultaneously and adaptability can be introduced to enhance their robustness. Predominantly single-input-single-output (SISO) and multi-SISO collocated sensor and actuator pairs have been utilized in direct output feedback schemes, for example, with DVF. Some recent AVC studies in this area of research have extended this work to include model-based control approaches, for example, pole-placement, which demonstrate increased flexibility in achieving desired vibration mitigation performances but for which stability and robustness issues must be adequately addressed. For example, pole-placement design via an algebraic approach is found to suffer from numerical difficulties as a result of rank deficiency with higher plant orders ${ }^{[7]}$.

Further author information:

DSN* Email: d.nyawako@sheffield.ac.uk, PR Email: p.reynolds@sheffield.ac.uk, p.reynolds@,fullscaledynamics.com, MJH Email: m.j.hudson@,sheffield.ac.uk

Active and Passive Smart Structures and Integrated Systems 2013, edited by Henry A. Sodano, Proc. of SPIE Vol. 8688, 868820 - (c) 2013 SPIE · CCC code: $0277-786 \mathrm{X} / 13 / \$ 18$ doi: $10.1117 / 12.2010375$ 
A recent and attractive controller scheme that has been developed for trials in the marine and aerospace sectors for large scale structures or those exposed to harsh environments is a geometric design approach for harmonic or broadband control of remotely located vibration ${ }^{[8,9,10]}$. The concept behind this controller design approach is that for large scale structures or where the system environment is harsh, it may not be feasible to locate sensors where vibration attenuation is desired or this may be prohibitively expensive. The optimal control of local vibration may in turn result in an enhancement at remote locations. These researches therefore define a design freedom for reducing vibration both at local and remote points, and can be applied for either discrete frequency and/or broadband control and often involve an inversion of the local path plant dynamics.

The motivation for the work presented in this paper comes from the work in ${ }^{[8,9,10]}$. A similar challenge to that described in the previous paragraph is often seen in this research work where the presence of services and other facilities often hinder the siting of actuators and sensors at desired locations. In the work presented here, an approximate pole-placement controller scheme is derived via the inversion of the floor model dynamics and this is designed to achieve target closedloop performances. This design approach has been found to avoid the numerical difficulties associated with the algebraic pole-placement approach. The laboratory structure model is estimated by a modal expansion approach from the experimental modal analysis (EMA) point accelerance frequency response function (FRF) data and is minimum-phase. The vibration mitigation performances achieved, which are expressed as FRFs, are compared to that of the commonly used DVF control scheme with inner loop actuator compensation that has been extensively used in past AVC field trials. The work in this paper is organized as follows: section 2 introduces the plant and actuator dynamics and the compensators used in this work are described in section 3. Section 4 presents the results of analytical studies and experimental implementation and conclusions are highlighted in section 5.

\section{PLANT MODEL AND ACTUATOR DYNAMICS}

The laboratory structure used for the AVC studies is shown in figure 1. It is a simply-supported in-situ cast posttensioned slab strip with a span of $10.8 \mathrm{~m}$, width of $2.0 \mathrm{~m}$ and depth of $275 \mathrm{~mm}$. The dynamic properties for the AVC studies are evaluated from a point accelerance FRF test using a collocated sensor and actuator pair at location S1. An analytical model of the measured point accelerance FRF used for the controller designs and stability studies is obtained in the Laplace domain using the modal expansion approach. For the consideration of only a finite set of modes within a frequency band of interest, for example ' $n$ ' modes within the frequency bandwidth $0-100 \mathrm{~Hz}$, the plant model $G_{s}(s)$ can be approximated by Eq. $1 . s$ is a complex variable, $\mu_{i} \geq 0, \zeta_{i}$ and $\omega_{i}$ are the inverse of the modal mass, damping ratio and natural frequency associated with the $i^{\text {th }}$ mode of vibration. In this work ' $n=4$ '. A summary of the modal properties of the first four modes of vibration estimated from EMA are shown in Table 1. This floor model is minimum phase (MP).

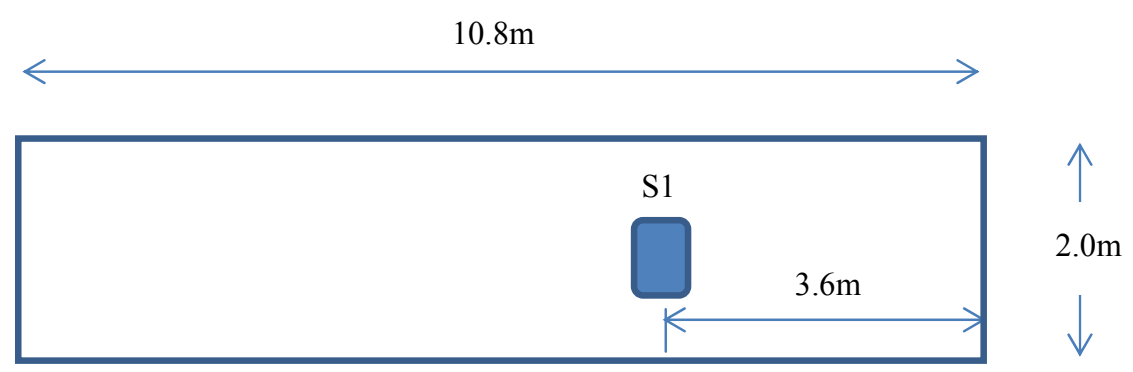

Figure 1. Plan view of laboratory structure showing test location for AVC studies

$G_{s}(s)=\sum_{i=1}^{4} \frac{\mu_{i} s^{2}}{s^{2}+2 \zeta_{i} \omega_{i} s+\omega_{i}^{2}}$ 
Table 1. Estimated modal properties of laboratory structure.

\begin{tabular}{|c|c|c|}
\hline Mode & $f_{i} \mathbf{( H z )}$ & $\zeta_{i}(\mathbf{\%})$ \\
\hline 1 & 4.45 & 0.7 \\
\hline 2 & 16.60 & 0.7 \\
\hline 3 & 63.30 & 4.4 \\
\hline 4 & 68.80 & 1.5 \\
\hline
\end{tabular}

The actuators are APS Dynamics model 400 electrodynamic shakers. They impart inertial forces into the structure on which they are placed. The dynamic characteristics of these shakers expressed by the TF between the force applied to the structure, $f(t)$, and the input voltage command, $v(t)$, can be described by the linear second order system in Eq. 2 when they are driven in the current drive mode, which is the mode of operation used here. $K_{c}=300, \zeta_{a c t}=0.07$ and $\omega_{a c t}=8.168 \mathrm{rads} / \mathrm{s}$ are the force-voltage constant, damping ratio and natural frequency of the actuators.

$G_{a c t}(s)=\frac{K_{c} s^{2}}{s^{2}+2 \zeta_{a c t} \omega_{a c t} s+\omega_{a c t}^{2}}$

Two actuators, one for excitation and the other for control, and a single sensor (Endevco 7754A-1000 piezoelectric accelerometer) were sited at location S1 in these studies.

\section{CONTROL SCHEMES}

The control schemes investigated in this research work are as follows:

a) Direct velocity feedback (DVF) with inner loop actuator compensation. This is implemented using a collocated sensor and actuator pair in a SISO scheme at location S1 in figure 1.

b) Approximate pole-placement controller with inversion in plant model. This is also implemented using a collocated sensor and actuator pair in a SISO scheme at location S1 in figure 1. Two compensators are designed here, one to isolate and control only the dominant mode of vibration and another to control both the two lowest modes of vibration.

\subsection{DVF Scheme}

DVF with inner loop compensation for the actuator is shown in figure 2. $C_{0}(s)$ is a lossy integrator that includes a feedback gain component, $\gamma_{d c}$, as shown in Eq. 3. This is designed to impart significant damping in the structure. The designed inner loop actuator compensator, $C_{I}(s)$, is shown in Eq. 4. This achieves a closed-loop transfer function of the actuator inner loop with a frequency of $1.30 \mathrm{~Hz}$ and a damping ratio of 0.60 . As noted before, DVF is implemented in a SISO set-up with the collocated actuator and sensor pairs sited at S1 in figure $1 . \gamma_{d c}=400$ and the DVF scheme is designed to achieve damping ratios of 0.19 and 0.06 in modes 1 and 2, and ensures a gain margin (GM) of $11.5 \mathrm{~dB}$ and phase margin (PM) greater than $30^{\circ}$. A second-order Butterworth filter with cut-off frequency $1.0-50 \mathrm{~Hz}$ is also implemented in both analytical and experimental studies. A typical root locus plot of the closed-loop system is shown in figure 3. 


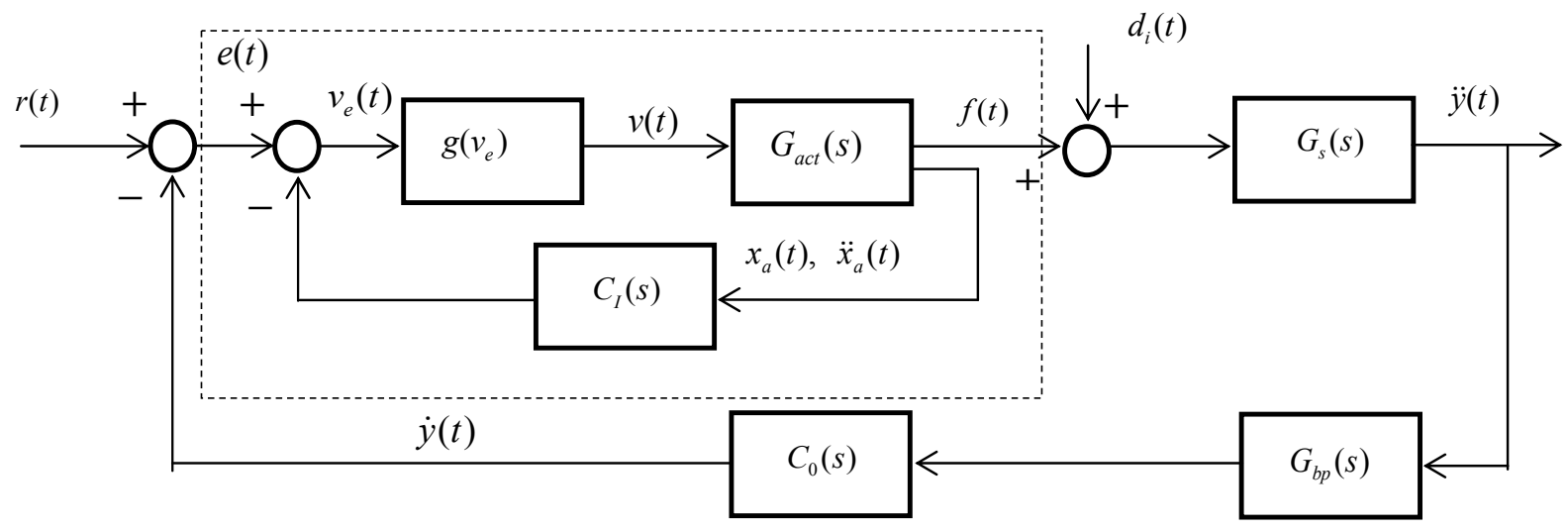

Figure 2. Direct velocity feedback with inner loop actuator compensation

Where:

$G_{s}(s) \quad$ Floor model

$G_{a c t}(s) \quad$ Actuator model

$G_{b p}(s) \quad$ Band pass filter ( $2^{\text {nd }}$ order Butterworth)

$C_{0}(s) \quad$ Transfer function of outer loop

$C_{I}(s) \quad$ Transfer function of inner loop

$x_{a}(t) \quad$ Displacement of actuator moving mass

$\ddot{x}_{a}(t) \quad$ Acceleration of actuator moving mass

$r(t) \quad$ Reference signal $\ddot{y}(t) \quad$ Structural acceleration response

$\dot{y}(t) \quad$ Structural velocity response

$f(t) \quad$ Actuator force

$v(t) \quad$ Final control voltage signal

$v_{e}(t) \quad$ Initial control voltage signal

$d_{i}(t) \quad$ Input disturbance

$e(t) \quad$ Error signal

$g\left(v_{e}\right) \quad$ Saturation nonlinearity

$C_{0}(s)=\frac{\gamma_{d c}}{s+\beta}, \quad C_{I}(s)=\frac{s^{2}+1.14 s+66.72}{s^{2}+9.80 s+66.72}$

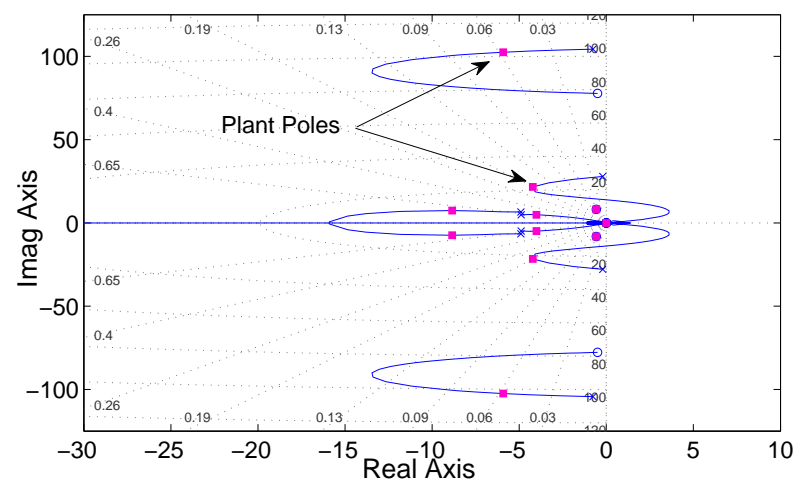

Figure 3. Root locus plot for DVF control scheme 


\subsection{Approximate pole-placement controller}

The approximate pole-placement controller in this work, making use of the minimum phase plant dynamics in Eq. 1, is derived partly from the work in $[8,9,10]$. In these works, the compensators are selected to achieve certain desired closedloop performances based on the inversion in the plant dynamics and the specification of some design variables. These past works have also been extended to deal with stable controller designs for non-minimum phase dynamics. From the works in $[8,9,10]$, the transfer function matrix relating the input to the output is formulated as shown in Eq. $5 . y(s)$ and $z(s)$ are the locally measured and remote vibration, respectively. $u(s)$ and $d(s)$ are the local control force and remote disturbance force. Eq. 6 shows the control input as a function of the locally measured vibration.

$\left[\begin{array}{l}y(s) \\ z(s)\end{array}\right]=\left[\begin{array}{ll}g_{11}(s) & g_{12}(s) \\ g_{21}(s) & g_{22}(s)\end{array}\right]\left[\begin{array}{l}u(s) \\ d(s)\end{array}\right]$

$u(s)=-k(s) y(s)$

Through the definition of a design variable, $\alpha(s)$ in Eq. 7, the location vibration output, $y(s)$ and remote vibration output, $z(s)$, in the presence of the feedback control signal in Eq. 6 can be expressed as shown in Eqs. 8 and 9.

$$
\begin{aligned}
& \alpha(s)=-\frac{g_{11}(s) k(s)}{1+g_{11}(s) k(s)} \\
& y(s)=[1+\alpha(s)] g_{12}(s) d(s) \\
& z(s)=\left[1+\alpha(s) \frac{g_{12}(s) g_{21}(s)}{g_{11}(s) g_{22}(s)}\right] g_{22}(s) d(s)
\end{aligned}
$$

In the present work, the excitation input is assumed to be collocated with the actuator-sensor pair used for control and Eqs. 8 and 9 are thereby identical. Thus, $g_{12}(s)=g_{21}(s)=g_{11}(s)=g_{22}(s)$. Eq. 8 can thus be expressed as shown in Eq. 10 in which $g_{11 \_d}(s)$ is the desired closed-loop dynamics of the floor. The compensator, $k(s)$, required to achieve the desired closed-loop dynamics is then expressed as shown in Eq. 11. A gain term, $k_{g}$ is introduced to improve stability margins as shown in Eq. 12.

$$
\begin{aligned}
& y(s)=[1+\alpha(s)] g_{11}(s) d(s)=g_{11 \_d}(s) d(s) \\
& k(s)=\frac{g_{11}(s)-g_{11 \_d}(s)}{g_{11}(s) g_{11_{-} d}(s)} \\
& k(s)=k_{g} \frac{g_{11}(s)-g_{11 \_d}(s)}{g_{11}(s) g_{11 \_d}(s)}
\end{aligned}
$$

The controller scheme for the approximate pole-placement controller also takes the form of figure 2 , where $C_{o}(s)=k(s)$ and $C_{I}(s)=0$ i.e. no inner loop compensation is provided for the actuator dynamics. A third-order Butterworth filter with cut-off frequency $1.5-30 \mathrm{~Hz}$ is implemented for controller scheme 1 that isolates and controls only the first mode of vibration of the laboratory structure, and a similar order filter with cut-off frequency $1.5-50 \mathrm{~Hz}$ is implemented for controller scheme 2 that targets both the first and second vibration modes of the laboratory structure. Two notch filters, $G_{n o t 1}(s)$ and $G_{n o t 2}(s)$ shown in Eqs. 13 and 14 are designed to attenuate resultant resonances from the compensator $k(s)$ due to the approximate plant inversion procedure. $k_{g}=0.0030,0.0015$ for controller schemes 1 and 2 , respectively. 
Typical root locus plots for the closed-loop system with the two sets of compensators designed here are shown in figures $4 \mathrm{a}$ and $4 \mathrm{~b}$. These are zoomed into the first two fundamental vibration modes of the laboratory structure for clarity.

$$
\begin{aligned}
& G_{n o t 1}(s)=\frac{s^{2}+1.15 s+3305}{s^{2}+2.30 s+3305} \\
& G_{n o t 2}(s)=\frac{s^{2}+50.27 s+6.317 e 004}{s^{2}+201.10 s+6.317 e 004}
\end{aligned}
$$

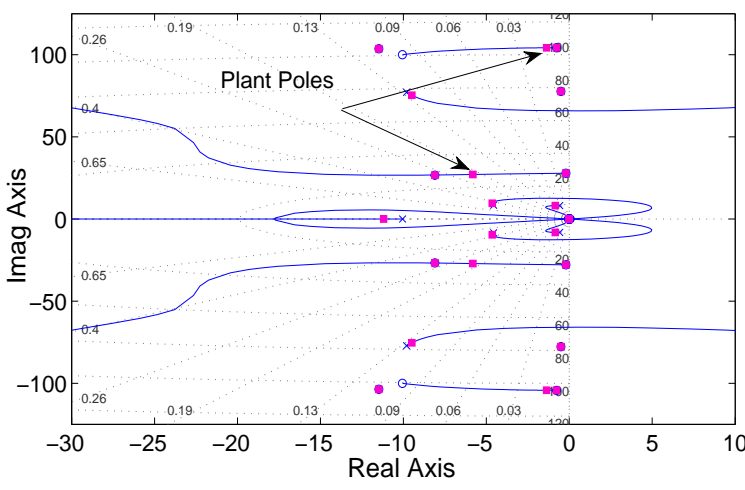

a)

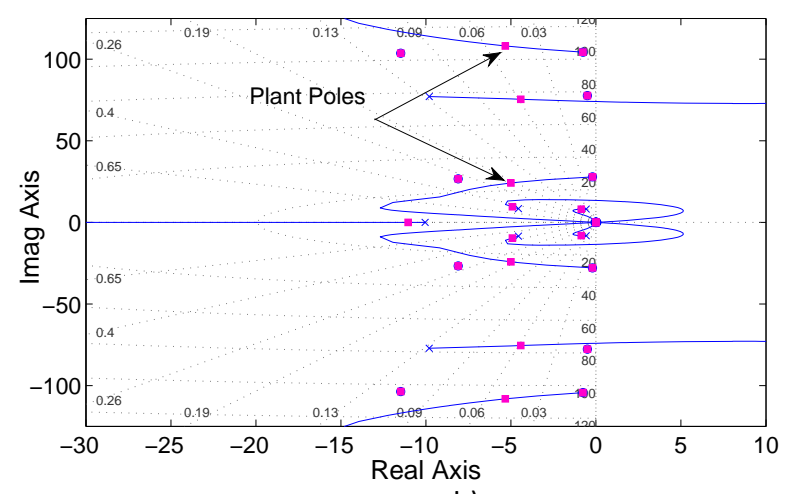

b)

Figure 4. Root locus plot for approximate PP type controller

\section{ANALYTICAL AND EXPERIMENTAL RESULTS}

For $s=j \omega$ and assuming a SDOF model of the laboratory structure based on its lowest mode of vibration as shown in Eq. 15, the compensator for the approximate pole-placement compensator in Eq. 13 can be expressed as shown in Eq. 16. Further, the integral term for DVF control scheme, with $\beta=0$ can be expressed as shown in Eq. 17. Assuming a target improvement in damping characteristic of the first mode of the laboratory structure from $\zeta_{1}=0.7 \%$ to $\zeta_{1} \_d=20.0 \%$, and with gain $k_{g}=0.002$ in Eq. 16, figure 5 shows that this compensator is identical to an ideal integrator with gain $\gamma_{d c}=400$ which in turn achieves the same function.

$$
\begin{aligned}
& g_{11}(j \omega)=\frac{-\mu_{1} \omega^{2}}{\left(\omega_{1}^{2}-\omega^{2}\right)+2 \zeta_{1} \omega_{1} \omega j} \\
& k(j \omega)=k_{g} \frac{g_{11}(j \omega)-g_{11_{\_} d}(j \omega)}{g_{11}(j \omega) g_{11_{-} d}(j \omega)} \\
& C_{o}(j \omega)=\frac{\gamma_{d c}}{j \omega}
\end{aligned}
$$




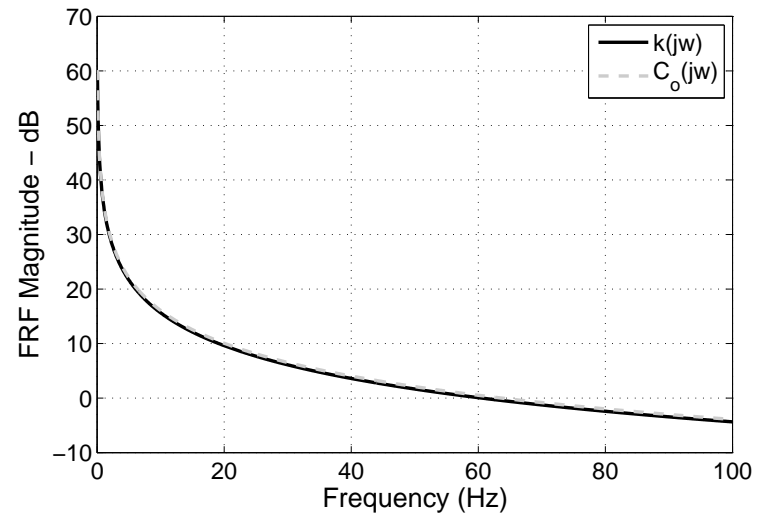

a) Magnitude Plot

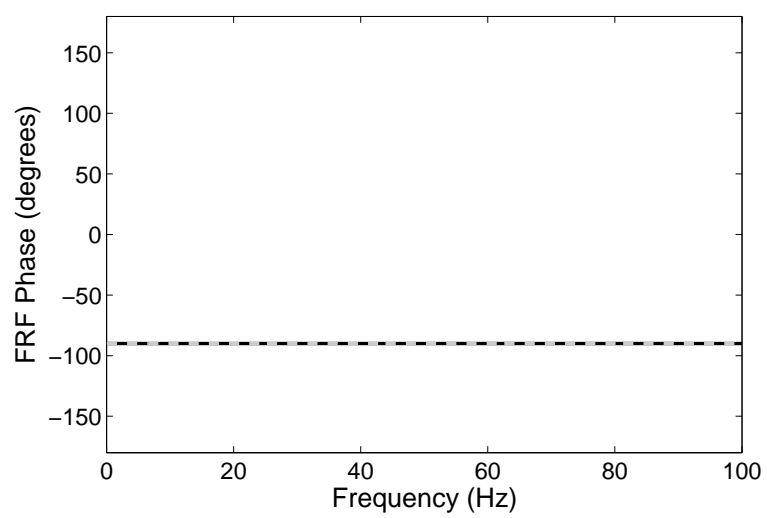

b) Phase Plot

Figure 5. Magnitude and phase plot for compensator, $k(j \omega)$, and ideal integrator, $C_{o}(j \omega)$

Figures $6 \mathrm{a}, 6 \mathrm{c}$ and $6 \mathrm{e}$ show the predicted analytical point accelerance FRFs for the three controllers investigated in this work, i.e. approximate pole-placement type controllers 1 and 2 and DVF control law. Also shown in Figures 6b, 6d and $6 \mathrm{f}$ are corresponding point accelerance FRFs from experimental implementation of the controllers at location S1 in figure 1 (and shown in figure 7). Vibration mitigation performances predicted in analytical studies are achieved in the experimental implementations. These designs realize attenuations of about $22 \mathrm{~dB}$ and $12 \mathrm{~dB}$ in the first and second modes of vibration. From Figs $6 \mathrm{c}$ and $6 \mathrm{~d}$, the gains have been lowered to minimize potential for instability from the resonant frequency around $10 \mathrm{~Hz}$, a feature that is seen in the root locus study plots in figure $4 \mathrm{~b}$. With the approximate pole-placement controller making use of an approximate inverse in the plant dynamics, there is flexibility to isolate and target specific modes of vibration as seen in figures $6 \mathrm{a}$ to $6 \mathrm{~d}$.

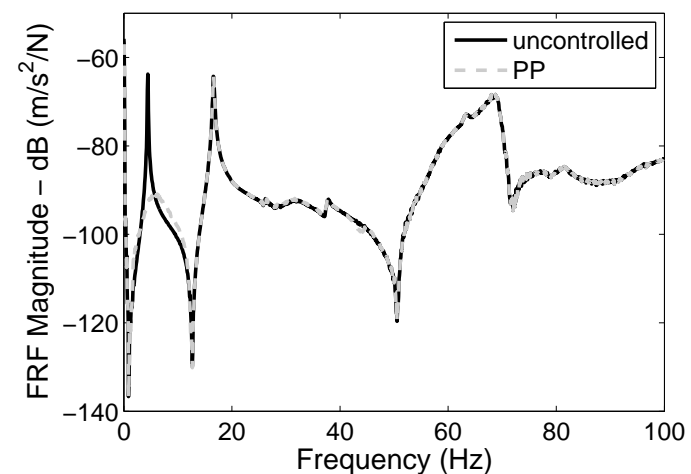

a) Analytical - PP type controller targeting mode 1 only

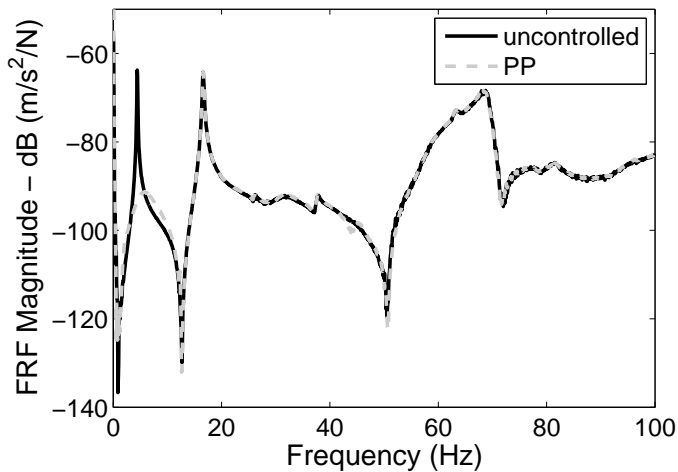

b) Experimental - PP type controller targeting mode 1 only 


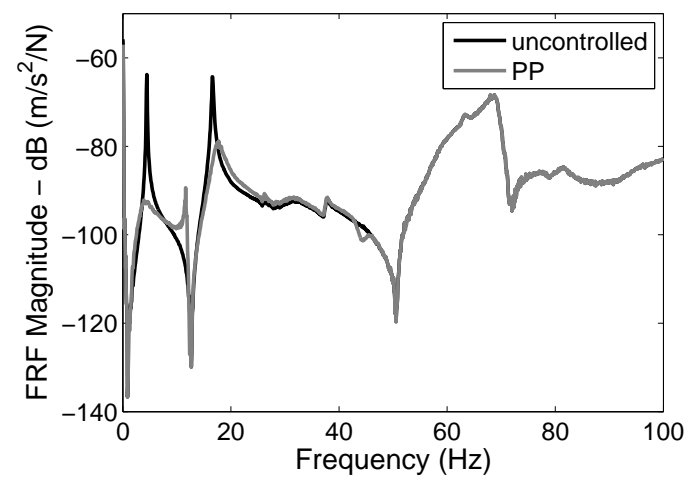

c) Analytical - PP type controller targeting modes 1 and 2

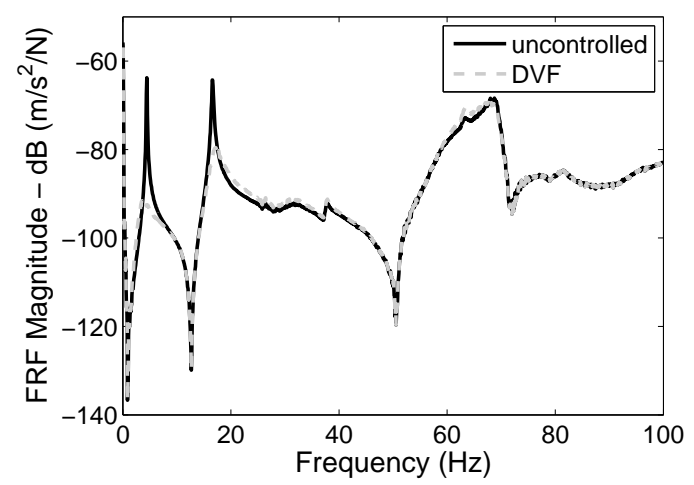

e) Analytical - DVF control law

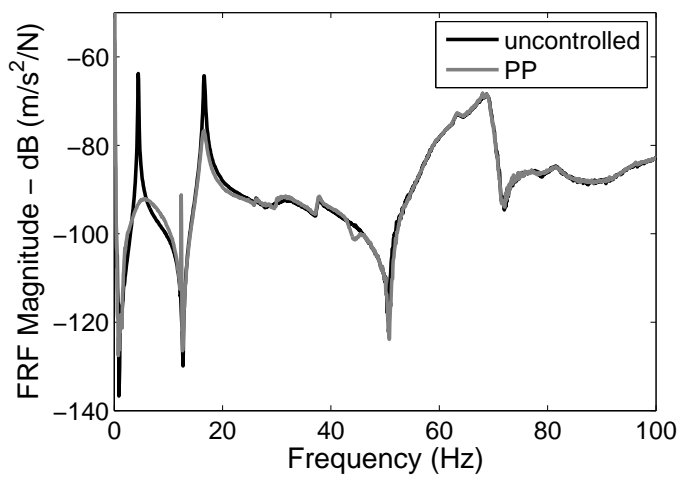

d) Experimental - PP type controller targeting modes 1 and 2

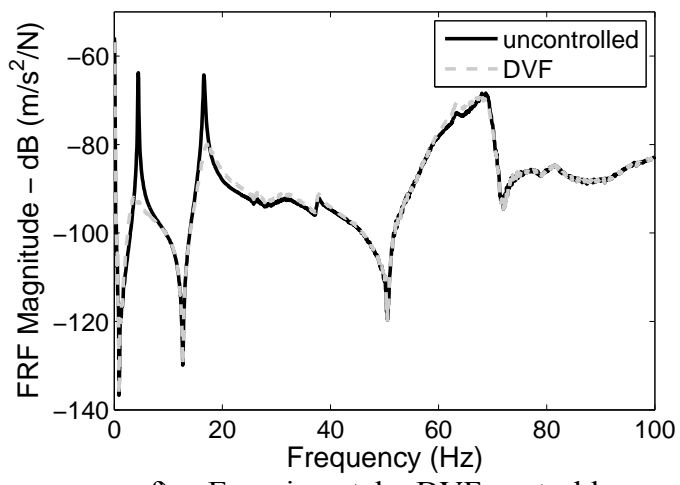

f) Experimental - DVF control law

Figure 6 Frequency response functions from analytical and experimental studies

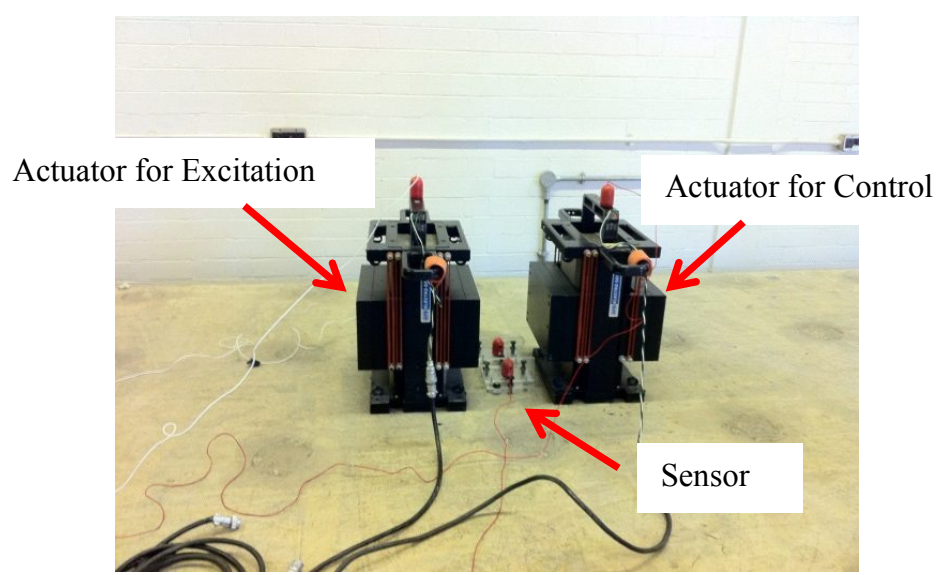

Figure 7. Experimental AVC studies at location S1 in figure 1 


\section{CONCLUSIONS}

This work shows results of some on-going AVC studies mainly focused on mitigation of human induced vibrations in problematic office floors. An approximate pole-placement type controller is derived based on inversion in plant dynamics and implemented in both analytical and experimental studies with appropriate compensation. The vibration mitigation performance of this controller scheme is compared to that of the DVF scheme with inner loop compensation for the actuators that has been widely used in this work.

Vibration mitigation performances between both approaches have been found to be comparable and the analytical predictions correlate well with the experimental results. A potential advantage of the approximate pole-placement type controller is the flexibility in its formulation since it can be designed to isolate and control target modes of vibration. Appropriate compensation in the form of notch filters is needed to attenuate potential resonances via the plant dynamics inversion process. In this work, controller 1 is designed to isolate and control only the dominant vibration mode whilst controller 2 has been designed to target both vibration modes. DVF which is quite robust targets both vibration modes.

The compensator derived via this approximate pole-placement controller has been found to default to an ideal integrator when a single degree of freedom plant model is used to realize it. In comparison to past studies on pole placement using an algebraic approach, numerical and stability issues as a result of rank deficiency are avoided.

\section{ACKNOWLEDGMENTS}

The authors would like to acknowledge the financial assistance provided by the UK Engineering and Physical Sciences Research Council (EPSRC) through a responsive mode grant entitled "Active Control of Human-Induced Vibration" (Ref: EP/H009825/1), Leadership Fellowship grant entitled "Advanced Technologies for Mitigation of Human-Induced Vibration" (Ref: EP/J004081/1) and Platform Grant entitled "Dynamic Performance of Large Civil Engineering Structures: An Integrated Approach to Management, Design and Assessment” (Ref: EP/G061130/1).

\section{REFERENCES}

[1] Reynolds, P., Diaz, I.M., and Nyawako, D.S., Vibration testing and active control of an office floor, In Proceedings of the 27th International Modal Analysis Conference, Orlando, Florida, USA (2009).

[2] Diaz, I.M., and Reynolds, P., Acceleration feedback control of human-induced floor vibrations, Engineering Structures 32, 163-173, (2010).

[3] Diaz, I.M., and Reynolds, P., Robust saturated control of human-induced floor Vibrations via a Proof-Mass Actuator, Smart Materials and Structures 18, 1-10, (2009).

[4] Nyawako, D.S., Reynolds, P., and Hudson, M.J., Findings with AVC design for mitigation of human induced vibrations in office floors, In Proceedings of the 31st International Modal Analysis Conference, Orange County, California, USA (2013).

[5] Diaz, I.M., Pereira E., and Reynolds P., Casado, C.M., Human-induced vibration cancellation using an active mass damper modified by an inner loop, Proceedings of the $8^{\text {th }}$ International Conference on Structural Dynamics, EURODYN 2011, Leuven, Belgium, 4-6 July, (2011).

[6] Nyawako, D., Diaz, I.M., Reynolds P., and Hudson, M., Enhancing velocity feedback with inner loop compensators for the actuators, SMART Conference 2011, Germany, 629-639, (2011).

[7] Chen, C.T., Linear system theory and design, Oxford University Press, ISBN 0-19-511777-8, (1999).

[8] Daley, S., Wang, J., A geometric approach to the design of remotely located vibration control systems, Journal of Sound and Vibration, 318, 702-714, (2008).

[9] Wang, J., Daley, S., Broad band controller design for remote vibration using a geometric approach, Journal of Sound and Vibration, 329, 3888-3897, (2010).

[10] Ubaid,U., Daley, S., Pope, S., Broadband design of remotely located vibration control systems : a stable solution for non-minimum phase dynamics, Inter-Noise 2011, Osaka, Japan, September 2011. 